\title{
Punding following posterior cerebral artery infarction: a case report and literature review
}

This article was published in the following Dove Press journal:

Neuropsychiatric Disease and Treatment

31 March 2017

Number of times this article has been viewed

\section{Cho-I Lin' \\ Yu-Ching Lin ${ }^{1-3}$ \\ Wei-Chih Lien' \\ Pei-Chun Hsieh' \\ Sheng-Han Lin' \\ Ta-Shen Kuan ${ }^{1,2}$}

'Department of Physical Medicine and Rehabilitation, National Cheng Kung University Hospital, College of Medicine, National Cheng Kung University, Tainan, Taiwan; ${ }^{2}$ Department of Physical Medicine and Rehabilitation, College of Medicine, National Cheng Kung University, Tainan, Taiwan; ${ }^{3}$ Medical Device Innovation Center, National Cheng Kung University, Tainan, Taiwan
Correspondence: Ta-Shen Kuan Department of Physical Medicine and Rehabilitation, National Cheng Kung University Hospital, College of Medicine, National Cheng Kung University, 138 Sheng-Li Road, Tainan 704, Taiwan Tel +886 62353535 ext 5242 Fax +886 62766106

Email kuan@mail.ncku.edu.tw
Introduction: Punding is a complex stereotyped behavior, characterized by excessiveness, non-goal orientation, and repetitiveness. It is mostly associated with Parkinson's disease, and very few cases in non-Parkinson's disease have been reported. We report a case of punding associated with supratentorial ischemic stroke.

Case presentation: We present a 70-year-old man with left posterior cerebral artery infarction with quetiapine-induced punding manifesting as repetitive unidirectional body turning. Remission of punding behavior ensued after cessation of quetiapine and administration of clonazepam.

Conclusion: This case describes the clinical course of quetiapine-induced punding in a patient with left posterior cerebral artery infarction. It suggests clonazepam may serve as a treatment option for poststroke punding.

Keywords: punding, stereotyped behavior, stroke, quetiapine, clonazepam

\section{Introduction}

Punding is a complex, stereotyped, prolonged, and non-goal oriented behavior, originally proposed by Rylander in 1972 to illustrate a unique repetitive behavior in illicit drug users. ${ }^{1}$ Although it was first noted in drug abusers,,${ }^{1,2}$ most cases of punding have been reported in patients with Parkinson's disease (PD). ${ }^{3}$ Only one case report delineated the development of punding in the subacute stage of pontine infarction. ${ }^{4}$ The spectrum of punding varies from as simple as hair brushing or paper shuffling, to more complicated activities such as hoarding, gardening, and tinkering with machinery. ${ }^{3}$ Cortico-striato-thalamo-cortical circuit has been postulated to be involved in the pathogenesis of punding, while its mechanism remains to be elucidated. ${ }^{3}$ Here we report a case of punding following left posterior cerebral artery infarction manifesting as repetitive unidirectional body turning. The condition persisted for 19 days and subsided after the administration of clonazepam.

\section{Case presentation}

A 70-year-old right-handed man was presented to our emergency room (ER) with acute onset of incoherent speech. His medical history was significant for hypertension and dyslipidemia even though he was not on antihypertensive, hypolipidemic nor other agents. The patient was a retired train operator and remained independent in activities of daily living until this episode. On examination, he was noted to have Wernicke's aphasia. There was no other cranial nerve palsy. Muscle strength of four extremities scored 5/5 according to the Medical Research Council (MRC) scale. Unenhanced computed tomography (CT) upon his arrival to the ER and magnetic resonance imaging $(\mathrm{MRI})$ of the brain (Figure 1) 2 days later confirmed the diagnosis 

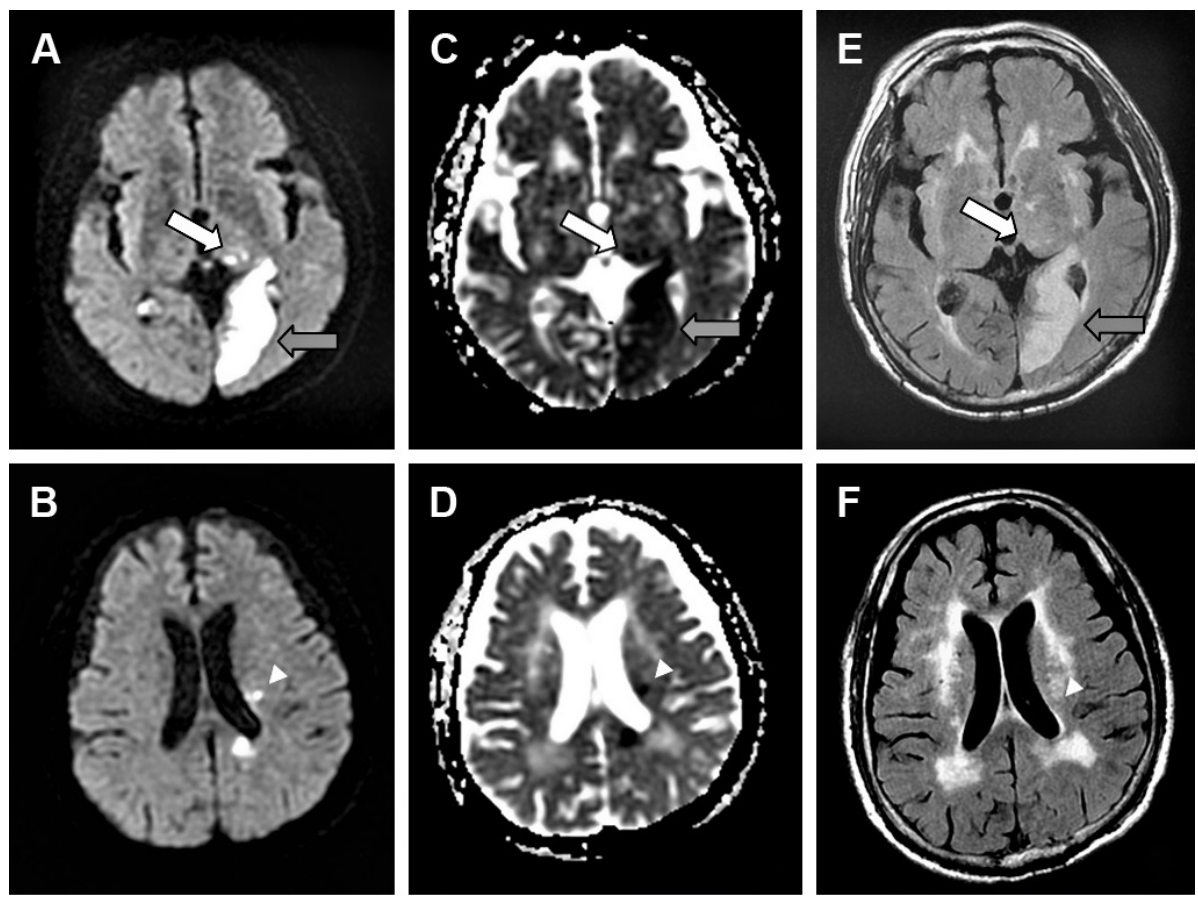

Figure I Magnetic resonance imaging results.

Notes: Hyperintensities noted in the left occipital lobe, corona radiate, and thalamus on transaxial diffusion weighted magnetic resonance imaging (A, B), and corresponding hypointensities on apparent diffusion coefficient map (C, D) suggested restricted diffusion resulting from an acute ischemic insult. Standard transaxial T2-weighted fluid attenuation inversion recovery sequence indicated hyperintensities in the corresponding region (E, F). Gray arrow, left occipital lobe; white arrow head, corona radiata; white arrow, thalamus.

of acute infarction in the territory of the left posterior cerebral artery, involving left occipito-temporo-parietal lobe, corona radiata, and thalamus. He was admitted to the neurology ward on the same day as the ER visit. His stroke deteriorated and he became hemiplegic on the right side with MRC score of $0 / 54$ days after admission. His condition improved 2 days later, after hydration.

Ten days after the stroke, the patient was transferred to a rehabilitation ward. During an interview with a physiatrist, he was noted to have transcortical sensory aphasia and right-sided hemiparesis with MRC score of 3/5. Behavior during the initial phase of rehabilitation was characterized by agitation, reluctance, and occasional episodes of yelling. He started on quetiapine $50 \mathrm{mg}$ at bedtime for intermittent irritability and poor sleep without concurrent administration of other hypnotics or dopaminergic medications. Eight days after initiation of quetiapine and 22 days after the stroke, he exerted unidirectional turning of his whole body to his right by $90^{\circ}$ in a clockwise fashion repetitively. Such behavior could last 6-8 hours during daytime and the patient appeared to be preoccupied. It could be interrupted temporarily by gesture command, and would subside while he slept. He became furious when asked to stop his activities. Electroencephalography at that time indicated no epileptiform discharge. He started on oral clonazepam
( $0.25 \mathrm{mg}$ daily at first, gradually up-titrated to $0.5 \mathrm{mg}$ twice daily within 1 week) 2 days after the onset of such behavior, and quetiapine was discontinued 2 days later. A gradual reduction in the duration of the stereotyped behavior was noted, and total remission was achieved 17 days after initiation of clonazepam therapy, and 15 days after cessation of quetiapine. The dosage of clonazepam was tapered off over a 1-week period (from $0.5 \mathrm{mg}$ twice daily to $0.5 \mathrm{mg}$ daily for the first 4 days, then $0.25 \mathrm{mg}$ daily for another 3 days, and then discontinued). There was no recurrence of punding in the following 6 months of follow-up.

Written informed consent was obtained from the patient and his guardians for publication of the case details, and any accompanying images.

\section{Discussion}

Punding is a complex stereotyped behavior featured by intense fascination with repetitive, excessive, non-goal oriented behavior. ${ }^{3,4}$ It is usually executed at the expense of other motor activities. ${ }^{3,5,6}$ The essential elements in punding include excessiveness, disruptiveness, and interruption resulting in anger. ${ }^{3,4}$ Patients with punding tend to be preoccupied by their chosen activities and withdraw into themselves, and this interferes with their interactions with the outer world. ${ }^{3-5}$ The above-mentioned characteristics of punding were all present 
in our case. The behavior pattern in our patient manifested as repetitive unidirectional body turning to the paretic side. Another possible explanation for such phenomenon was clonic perseveration, in which the inappropriate repetition of an action persisted despite absence of an ongoing cue. However, the phenomenon observed in our case was not triggered by external cues, and that made clonic perseveration a less likely explanation.

Most cases of punding have been reported in patients with PD. ${ }^{3}$ Observation of patients with PD suggests the spectrum of behavior pattern is influenced by gender, occupation, or even hobbies. ${ }^{3}$ However, such associations are not noted in our case. The behavior pattern of punding ranges from simple form such as repetitive hair brushing and repeatedly opening and closing handbags, to complex form like gardening. ${ }^{3}$ There is only one case report in the literature describing the emergence of punding following brainstem infarction. ${ }^{4}$ Moreover, very few cases of stroke-induced stereotyped behavior have been reported. Available PubMed literature retrieved on January 19, 2017 by searching for "stereotypy", "stereotyped behavior", and "stroke" has been summarized in Table 1, and additional relevant case reports referenced by these publications are also included. Our review suggests basal ganglion and thalamus (two out of seven cases, respectively) are the most frequently involved locations, and the others include pons, cerebellum, frontal and parietal lobes. The mean latency from onset of cerebrovascular events to the occurrence of stereotyped behavior is 38.6 days (ranging from 5 to 90 days). In our case, the acute infarction in the territory of left posterior cerebral artery mainly involved the left occipital lobe and left thalamus. The occipital lobe is known for vision processing but has not been reported to be associated with stereotyped behavior. In contrast, there were two case reports signifying the involvement of thalamus in the development of poststroke stereotyped behavior. ${ }^{8,9}$

Although the underlying pathophysiology of punding remains uncertain, it has been attributed to excessive dopaminergic stimulation. ${ }^{3}$ Indeed, most punding behavior is observed in levodopa-treated PD patients, and reduction of the dose of antiparkinsonian medications results in improvement in punding behavior. ${ }^{3}$ Other medications, aside from dopaminergic replacement therapy, may play a relevant role in development of punding., ${ }^{3,4,10}$ Miwa et al reported emergence of punding after starting quetiapine therapy in two PD patients, the dosing of quetiapine ranged from 100-200 mg per day. ${ }^{10}$ Quetiapine is an atypical antipsychotic drug that interacts with multiple neurotransmitter receptors, with a higher affinity for serotonin receptors (5-hydroxytryptamine 2, 5-HT2) than its dopamine counterpart (D1 or D2). ${ }^{3,10}$ It has been shown to induce dopamine release by serotonin 5-HT2 receptor blockade. ${ }^{3}$ In our case, although the dose of quetiapine was relatively low, the temporal relationship of quetiapine initiation and punding occurrence, as well as its cessation and subsidence of punding supports a reciprocal link between quetiapine and punding. Furthermore, as infarction in posterior cerebral artery territory, especially the involvement of thalamus, disrupted the cortico-striato-thalamo-cortical pathway, the administration of quetiapine might exert a synergistic effect and both play pivotal roles in the development of punding in our case.

Cortico-striato-thalamo-cortical circuits that originate in the prefrontal cortex and project to the striatum and thalamus, and then return to prefrontal cortex, have been proven to play a vital role in the pathogenesis of behavioral and movement disorders. ${ }^{8,9,11}$ Theoretically, reduced basal ganglia output would result in disinhibition of the corticostriato-thalamo-cortical circuits, exciting cortical motor areas to exhibit movements that ought to be suppressed.,12 Gamma-aminobutyric acid (GABA) has been shown to be a vital neurotransmitter in the basal ganglia output pathway. ${ }^{12,13}$ Clonazepam is a benzodiazepine and acts as an enhancer of GABA-mediated effects. ${ }^{12,13}$ As a consequence, it might increase GABAergic neurotransmission and thereby modulate movement disorders. ${ }^{6,12}$ In clinical practice, clonazepam has been proven to be effective in several poststroke movement disorders including hemichorea, hemiballism, dystonia, myoclonus, and tremor. ${ }^{13,14}$

To the best of our knowledge, complex stereotyped behavior resulting from cerebrovascular accidents is very rare in the existing literature. We reported the first case depicting the occurrence of punding following supratentorial ischemic stroke. Punding greatly impeded rehabilitation participation and endangered social interaction in our case, and that is a common issue among other patients with punding. $3,10,15,16$ Considering the wide application of clonazepam and its proven effectiveness in poststroke movement disorder, ${ }^{12,13}$ we treated this patient with clonazepam dosed as $0.5 \mathrm{mg}$ twice a day. Punding remitted thoroughly after 17 days of clonazepam therapy, and there was no recurrence of punding after discontinuation of clonazepam in the following 6 months. The therapeutic effect of clonazepam appeared to be optimal in our patient. It warrants further investigation to provide more evidence of clonazepam for treatment of poststroke punding. 







\section{Conclusion}

This case report presented the clinical course of quetiapineinduced punding in a patient with left posterior cerebral artery infarction. We also described the effectiveness of clonazepam for punding. Although there is no standard treatment, clonazepam may be a treatment option for poststroke punding.

\section{Acknowledgments}

This article was supported by a research grant from National Cheng Kung University Hospital (NCKUH-10605013), College of Medicine, National Cheng Kung University, Tainan, Taiwan.

\section{Disclosure}

The authors report no conflicts of interest in this work.

\section{References}

1. Rylander G. Psychoses and the punding and choreiform syndromes in addiction to central stimulant drugs. Psychiatr Neurol Neurochir. 1972;75(3):203-212.

2. Schiorring E. Psychopathology induced by "speed drugs". Pharmacol Biochem Behav. 1981;14 Suppl 1:109-122.

3. Fasano A, Petrovic I. Insights into pathophysiology of punding reveal possible treatment strategies. Mol Psychiatry. 2010;15(6):560-573.

4. Nguyen FN, Pauly RR, Okun MS, Fernandez HH. Punding as a complication of brain stem stroke?: report of a case. Stroke. 2007;38(4): $1390-1392$.
5. Fasano A, Evans AH. Is punding a stereotypy? Mov Disord. 2013; 28(3):404-405.

6. Mehanna R, Jankovic J. Movement disorders in cerebrovascular disease. Lancet Neurol. 2013;12(6):597-608.

7. Fung VS, Morris JG, Leicester J, Soo YS, Davies L. Clonic perseveration following thalamofrontal disconnection: a distinctive movement disorder. Mov Disord. 1997;12(3):378-385.

8. Arauco AR, Grados MA, Vizcarra D. A diffuse brain injury leading to a complex neurobehavioral syndrome. J Neuropsychiatry Clin Neurosci. 2008;20(2):240-241.

9. Pandey S, Sarma N. Stereotypy after acute thalamic infarct. JAMA Neurol. 2015;72(9):1068.

10. Miwa H, Morita S, Nakanishi I, Kondo T. Stereotyped behaviors or punding after quetiapine administration in Parkinson's disease. Parkinsonism Relat Disord. 2004;10(3):177-180.

11. Herrero MT, Barcia C, Navarro JM. Functional anatomy of thalamus and basal ganglia. Childs Nerv Syst. 2002;18(8):386-404.

12. Siniscalchi A, Gallelli L, De Sarro G. Use of antiepileptic drugs for hyperkinetic movement disorders. Curr Neuropharmacol. 2010; 8(4):359-366.

13. Siniscalchi A, Gallelli L, Labate A, Malferrari G, Palleria C, Sarro GD. Post-stroke movement disorders: clinical manifestations and pharmacological management. Curr Neuropharmacol. 2012;10(3):254-262.

14. Nakawah MO, Lai EC. Post-stroke dyskinesias. Neuropsychiatr Dis Treat. 2016;12:2885-2893.

15. McGrath CM, Kennedy RE, Hoye W, Yablon SA. Stereotypic movement disorder after acquired brain injury. Brain Inj. 2002;16(5):447-451.

16. Maraganore DM, Lees AJ, Marsden CD. Complex stereotypies after right putaminal infarction: a case report. Mov Disord. 1991;6(4):358-361.

17. Kulisevsky J, Berthier ML, Avila A, Roig C. Unilateral parkinsonism and stereotyped movements following a right lenticular infarction. Mov Disord. 1996;11(6):752-754.

18. Lee D, Lee D, Ahn TB. Stereotypy after cerebellar infarction. J Neurol Sci. 2014;344(1-2):227-228.
Neuropsychiatric Disease and Treatment

\section{Publish your work in this journal}

Neuropsychiatric Disease and Treatment is an international, peerreviewed journal of clinical therapeutics and pharmacology focusing on concise rapid reporting of clinical or pre-clinical studies on a range of neuropsychiatric and neurological disorders. This journal is indexed on PubMed Central, the 'PsycINFO' database and CAS,

\section{Dovepress}

and is the official journal of The International Neuropsychiatric Association (INA). The manuscript management system is completely online and includes a very quick and fair peer-review system, which is all easy to use. Visit http://www.dovepress.com/testimonials.php to read real quotes from published authors. 